\title{
Existence of positive solutions for Caputo fractional difference equation
}

\author{
Huiqin Chen ${ }^{1}$, Zhen $\mathrm{Jin}^{2}$ and Shugui Kang ${ }^{1 *}$
}

"Correspondence:
dtkangshugui@126.com
'School of Mathematics and
Computer Sciences, Shanxi Datong
University, Datong, Shanxi 037009,
P.R. China
Full list of author information is
available at the end of the article

available at the end of the article

\begin{abstract}
In this paper, we studied the following Caputo fractional difference boundary value problem (FBVP): $\Delta_{C}^{v} y(t)=-f(t+v-1, y(t+v-1)), y(v-3)=\Delta y(b+v)=\Delta^{2} y(v-3)=0$, where $2<v \leq 3$ is a real number, $\Delta_{C}^{v} y(t)$ is the standard Caputo difference. By means of cone theoretic fixed point theorems, some results on the existence of one or more positive solutions for the above Caputo fractional boundary value problems are obtained.
\end{abstract}

MSC: 26A33; 39A05; 39A12

Keywords: Caputo fractional difference; boundary value problem; Green's function; existence of positive solution

\section{Introduction}

In this paper, we discuss the following Caputo fractional difference boundary value problem (FBVP):

$$
\left\{\begin{array}{l}
\triangle_{C}^{v} y(t)=-f(t+v-1, y(t+v-1)), \\
y(v-3)=\triangle y(b+v)=\triangle^{2} y(v-3)=0,
\end{array}\right.
$$

where $t \in[0, b+1]_{\mathbb{N}_{0}}, b \geq 5$ is an integer. $f:[v-2, v-1, \ldots, b+v]_{\mathbb{N}_{v-2}} \times[0,+\infty) \rightarrow[0,+\infty)$ is continuous and $f$ is not identically zero, $2<v \leq 3$, and $\triangle_{C}^{v} y(t)$ is the standard Caputo difference.

Fractional difference equations appear in useful biological models [1]. Influenced by the results of [1-3], the existence of one or two positive solutions for fractional difference equations has been studied (see [4-14]). Among them, in [4, 5], the authors introduced the fractional sum and difference operators, studied their behavior and developed a complete theory governing their compositions. In [6], Atici and Eloe studied the following two-point boundary value problem for fractional difference equation:

$$
\left\{\begin{array}{l}
-\triangle^{v} y(t)=f(t+v-1, y(t+v-1)) \\
y(v-2)=0, \quad y(b+v+1)=0
\end{array}\right.
$$

where $1<v \leq 2$. They obtained the existence of positive solutions by means of the Krasnosel'skii fixed point theorem. Goodrich gave some new existence results for (2) in [7].

\section{Springer}

(c) 2015 Chen et al.; licensee Springer. This is an Open Access article distributed under the terms of the Creative Commons Attribution License (http://creativecommons.org/licenses/by/4.0), which permits unrestricted use, distribution, and reproduction in any medium, provided the original work is properly credited. 
Goodrich [8] also considered the following fractional boundary value problem with nonlocal conditions:

$$
\left\{\begin{array}{l}
-\triangle^{v} y(t)=f(t+v-1, y(t+v-1)) \\
y(v-2)=g(y), \quad y(b+v+1)=0
\end{array}\right.
$$

where $1<v \leq 2$. He showed a uniqueness theorem by means of the contraction and an existence theorem by means of the Brouwer theorem for (3). He also presented the existence of at least one positive solution to (3) by using the Krasnosel'skii theorem. In [912], the authors deduced the existence of one or more positive solutions for fractional difference equations. Recently, $\mathrm{Wu}$ and Baleanu introduced some applications of the Caputo fractional difference to discrete chaotic maps in $[13,14]$. However, to the best of our knowledge, few papers can be found in the literature dealing with the Caputo fractional difference equation boundary value problems. Motivated by [15], we make some attempt to fill this gap in the existing literature in this paper. We consider the existence of one or more positive solutions for the boundary value problem of the Caputo fractional difference equation (1).

The article is structured as follows. In Section 2, we deduce the Green's function of the FBVP (1). Then we prove that Green's function satisfies the useful properties. In Section 3, we present and prove our main results. In Section 4, we give some examples to illustrate our results.

\section{The Green's function}

We first review some basic results about fractional sums and differences. For any $t \in[0$, $b+1]_{\mathbb{N}_{0}}$ and $v>0$, we define

$$
t^{\underline{v}}=\frac{\Gamma(t+1)}{\Gamma(t+1-v)}
$$

for which the right-hand side is defined. We appeal to the convention that if $t+1-v$ is a pole of the Gamma function and $t+1$ is not a pole, then $t^{\underline{v}}=0$.

The $v$ th fractional sum of a function $f$ is defined by

$$
\triangle^{-v} f(t)=\frac{1}{\Gamma(v)} \sum_{s=a}^{t-v}(t-s-1)^{\frac{v-1}{f}} f(s)
$$

for $v>0$ and $t \in\{a+v, a+v+1, \ldots\}=\mathbb{N}_{a+v}$. We also define the $v$ th Caputo fractional difference for $v>0$ by

$$
\triangle_{C}^{v} f(t)=\Delta^{-(n-v)} \triangle^{n} f(t)=\frac{1}{\Gamma(n-v)} \sum_{s=a}^{t-(n-v)}(t-s-1)^{\frac{n-v-1}{}} \triangle_{a}^{n} f(s),
$$

where $n-1<v \leq n$.

First, we give the following two lemmas, they can be found in the recent papers (see, e.g., $[4,5])$. 
Lemma 2.1 [4] Assume that $v>0$ and $f$ is defined on domains $\mathbb{N}_{a}$, then

$$
\triangle_{a+(n-v)}^{-v} \Delta_{C}^{v} f(t)=f(t)-\sum_{k=0}^{n-1} c_{k}(t-a)^{\underline{k}}
$$

where $c_{k} \in \mathbb{R}, k=0,1, \ldots n-1$, and $n-1<v \leq n$.

Lemma 2.2 [5] Let $f: \mathbb{N}_{a+v} \times \mathbb{N}_{a} \rightarrow \mathbb{R}$ be given. Then

$$
\triangle\left(\sum_{s=a}^{t-v} f(t, s)\right)=\sum_{s=a}^{t-v} \triangle_{t} f(t, s)+f(t+1, t+1-v), \quad \text { for } t \in \mathbb{N}_{a+v} .
$$

In order to get our main results, we first give an important lemmas. This lemma will give a representation for the solution of (1), provided that the solution exists.

Lemma 2.3 Let $2<v \leq 3$ and $g:[v-2, v-1, \ldots, b+v]_{\mathbb{N}_{v-2}} \rightarrow \mathbb{R}$ be given. Then the solution of the FBVP

$$
\left\{\begin{array}{l}
\triangle_{C}^{v} y(t)=-g(t+v-1) \\
y(v-3)=\Delta y(b+v)=\Delta^{2} y(v-3)=0
\end{array}\right.
$$

is given by

$$
y(t)=\sum_{s=0}^{b+1} G(t, s) g(s+v-1)
$$

where the Green's function $G:[v-2, v-1, \ldots, b+v]_{\mathbb{N}_{v-2}} \times[0, b+1]_{\mathbb{N}_{0}} \rightarrow \mathbb{R}$ is defined by

$$
G(t, s)=\frac{1}{\Gamma(v)}\left\{\begin{array}{l}
(v-1)(t-v+3)(b+v-s-1)^{\frac{v-2}{}}-(t-s-1)^{\frac{v-1}{},} \\
0 \leq s<t-v+1 \leq b+1, \\
(v-1)(t-v+3)(b+v-s-1)^{\frac{v-2}{2},} \quad 0 \leq t-v+1 \leq s \leq b+1 .
\end{array}\right.
$$

Proof We apply Lemma 2.1 to reduce (4) to an equivalent summation equation

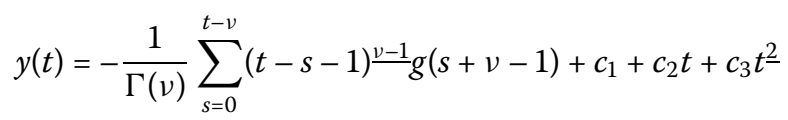

for some $c_{i} \in \mathbb{R}, i=1,2,3$. By Lemma 2.2, we have

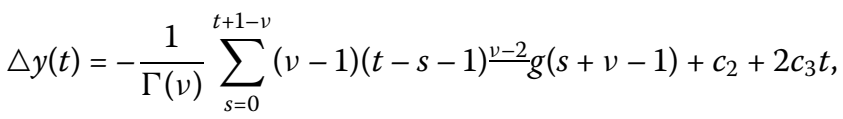

$$
\begin{aligned}
& \triangle^{2} y(t)=-\frac{1}{\Gamma(v)} \sum_{s=0}^{t+2-v}(v-1)(v-2)(t-s-1) \frac{v-3}{g} g(s+v-1)+2 c_{3} .
\end{aligned}
$$

From (4), we get

$$
c_{1}=-\frac{v-3}{\Gamma(v-1)} \sum_{s=0}^{b+1}(b+v-s-1)^{\frac{v-2}{g}} g(s+v-1),
$$




$$
c_{2}=\frac{1}{\Gamma(v-1)} \sum_{s=0}^{b+1}(b+v-s-1)^{\frac{v-2}{g} g(s+v-1), \quad c_{3}=0 .}
$$

Therefore, the solution of the FBVP (4) is

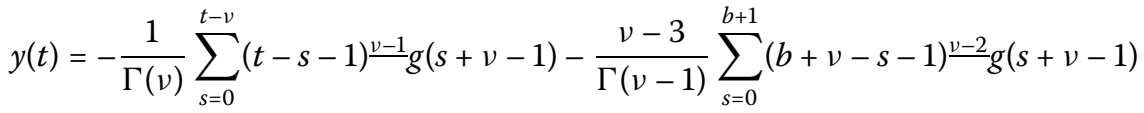

$$
\begin{aligned}
& +\frac{t}{\Gamma(v-1)} \sum_{s=0}^{b+1}(b+v-s-1)^{\frac{v-2}{g} g(s+v-1)} \\
& =\frac{1}{\Gamma(v)} \sum_{s=0}^{t-v}\left[(v-1)(t-v+3)(b+v-s-1)^{\frac{v-2}{2}}-(t-s-1)^{\frac{v-1}{2}}\right] g(s+v-1) \\
& +\frac{1}{\Gamma(v-1)} \sum_{s=t-v+1}^{b+1}(t-v+3)(b+v-s-1)^{\frac{v-2}{g} g(s+v-1)} \\
& =\sum_{s=0}^{b+1} G(t, s) g(s+v-1) \text {. }
\end{aligned}
$$

Remark Notice that $G(v-3, s)=0, G(t, b+2)=0$. $G$ could be extended to [ $v-3$, $b+v]_{\mathbb{N}_{v-3}} \times[0, b+2]_{\mathbb{N}_{0}}$, so we only discuss $(t, s) \in[v-2, b+v]_{\mathbb{N}_{v-2}} \times[0, b+1]_{\mathbb{N}_{0}}$.

Lemma 2.4 The Green's function $G$ has the following properties:

(i) $G(t, s)>0,(t, s) \in[v-2, b+v]_{\mathbb{N}_{v-2}} \times[0, b+1]_{\mathbb{N}_{0}}$.

(ii) $\max _{t \in[v-2, b+v]_{\mathbb{N}_{v-2}}} G(t, s)=G(b+v, s), s \in[0, b+1]_{\mathbb{N}_{0}}$.

(iii) $\min _{\frac{b+v}{4} \leq t \leq \frac{3(b+v)}{4}} G(t, s) \geq \frac{1}{4} \max _{t \in[v-2, b+v]_{\mathbb{N}_{v-2}}} G(t, s)=\frac{1}{4} G(b+v, s), s \in[0, b+1]_{\mathbb{N}_{0}}$.

Proof (i) From a representation of $G(t, s)$, it is clear that $G(t, s)>0$.

(ii) By a representation of $G(t, s)$, we have $\triangle_{t} G(t, s)>0$ for $0 \leq t-v+1 \leq s \leq b+1$ or $0 \leq s<t-v+1 \leq b+1$. Hence $G(t, s)$ is increasing to $t$ for $s \in[0, b+1]_{\mathbb{N}_{0}}$. From this we may conclude that

$$
\max _{t \in[v-2, v+b]_{\mathbb{N}_{v-2}}} G(t, s)=G(b+v, s), \quad s \in[0, b+1]_{\mathbb{N}_{0}} .
$$

(iii) Since

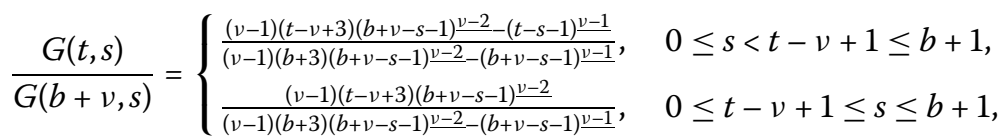

for $t-v+1 \leq s$ and $\frac{b+v}{4} \leq t \leq \frac{3(b+v)}{4}$, we get

$$
\frac{G(t, s)}{G(b+v, s)} \geq \frac{(v-1)(t-v+3)(b+v-s-1)^{\frac{v-2}{2}}}{(v-1)(b+3)(b+v-s-1)^{\frac{v-2}{2}}} \geq \frac{1}{4} .
$$

For $s<t-v+1$ and $\frac{b+v}{4} \leq t \leq \frac{3(b+v)}{4}$, we have

$$
\min _{\frac{b+v}{4} \leq t \leq \frac{3(b+v)}{4}} \frac{G(t, s)}{G(b+v, s)}=\frac{(v-1)\left(\frac{b+v}{4}-v+3\right)(b+v-s-1)^{\frac{v-2}{2}}-\left(\frac{b+v}{4}-s-1\right)^{\frac{v-1}{2}}}{(v-1)(b+3)(b+v-s-1) \frac{v-2}{2}-(b+v-s-1)^{\frac{v-1}{4}}} .
$$


Now, we want to get

$$
\frac{(v-1)\left(\frac{b+v}{4}-v+3\right)(b+v-s-1) \frac{v-2}{-}-\left(\frac{b+v}{4}-s-1\right) \frac{v-1}{2}}{(v-1)(b+3)(b+v-s-1) \frac{v-2}{4}-(b+v-s-1) \frac{1}{4}}
$$

that is,

$$
\begin{gathered}
(v-1)\left(\frac{b+v}{4}-v+3\right)(b+v-s-1) \frac{v-2}{4}-\left(\frac{b+v}{4}-s-1\right)^{\frac{v-1}{}} \\
\geq \frac{1}{4}(v-1)(b+3)(b+v-s-1) \frac{v-2}{4}-\frac{1}{4}(b+v-s-1)^{\frac{v-1}{}} .
\end{gathered}
$$

In fact

$$
\frac{b+v}{4}-v+3 \geq \frac{b+3}{4}, \quad \frac{\frac{1}{4}(b+v-s-1)^{\frac{v-1}{}}}{\left(\frac{b+v}{4}-s-1\right)^{\frac{v-1}{2}}} \geq 1 .
$$

So (5) holds, which completes the proof.

Lemma 2.5 Iff : $[v-2, v-1, \ldots, b+v]_{\mathbb{N}_{v-2}} \times[0,+\infty) \rightarrow[0, \infty)$ is a continuous function. Then the solutions y of the FBVP (1) satisfy

$$
\min _{\frac{b+v}{4} \leq t \leq \frac{3(b+v)}{4}} y(t) \geq \frac{1}{4} \max _{[v-2, b+v]_{\mathbb{N}_{\nu-2}}}|y(t)|
$$

Proof Taking into account Lemma 2.4, we have

$$
\begin{aligned}
\min _{\frac{b+v}{4} \leq t \leq \frac{3(b+v)}{4}}|y(t)| & \geq \frac{1}{4} \sum_{s=0}^{b+1} G(b+v, s) f(s+v-1, y(s+v-1)) \\
& \geq \frac{1}{4} \max _{t \in[v-2, b+v]_{\mathbb{N}_{\nu-2}}} \sum_{s=0}^{b+1} G(t, s) f(s+v-1, y(s+v-1)) \\
& =\frac{1}{4} \max _{[v-2, b+v]_{\mathbb{N}_{v-2}}}|y(t)| .
\end{aligned}
$$

The following fixed point theorem will play a major role in our main results.

Lemma 2.6 [16] Let $\mathcal{B}$ a Banach space and let $\mathcal{K} \subseteq \mathcal{B}$ be a cone. Assume that $\Omega_{1}$ and $\Omega_{2}$ are bounded open sets contained in $\mathcal{B}$ such that $0 \in \Omega_{1}$ and $\bar{\Omega}_{1} \subseteq \Omega_{2}$. Further, assume that $T: \mathcal{K} \cap\left(\bar{\Omega}_{2} \backslash \Omega_{1}\right) \rightarrow \mathcal{K}$ is a completely continuous operator. If either

(i) $\|T y\| \leq\|y\|$ for $y \in \mathcal{K} \cap \partial \Omega_{1}$ and $\|T y\| \geq\|y\|$ for $y \in \mathcal{K} \cap \partial \Omega_{2}$; or

(ii) $\|T y\| \geq\|y\|$ for $y \in \mathcal{K} \cap \partial \Omega_{1}$ and $\|T y\| \leq\|y\|$ for $y \in \mathcal{K} \cap \partial \Omega_{2}$.

Then the operator $T$ has at least one fixed point in $\mathcal{K} \cap\left(\bar{\Omega}_{2} \backslash \Omega_{1}\right)$.

\section{Main results}

For the sake of convenience, we denote

$$
f_{0}=\liminf _{y \rightarrow 0} \min _{t \in[v-2, b+v]_{\mathbb{N}_{\nu-2}}} \frac{f(t, y)}{y}, \quad f^{0}=\limsup _{y \rightarrow 0} \max _{t \in[v-2, b+v]_{\mathbb{N}_{v-2}}} \frac{f(t, y)}{y}
$$




$$
\begin{aligned}
& f_{\infty}=\liminf _{y \rightarrow+\infty} \min _{t \in[v-2, b+v]_{\mathbb{N}-2}} \frac{f(t, y)}{y}, \quad f^{\infty}=\limsup _{y \rightarrow+\infty} \max _{t \in[\nu-2, b+v]_{\mathbb{N}_{\nu-2}}} \frac{f(t, y)}{y}, \\
& \frac{1}{A}=\sum_{s=0}^{b+1} G(b+v, s), \quad \frac{1}{B}=\frac{1}{4} \sum_{s=\left[\frac{b+v}{4}-v+1\right]}^{\left[\frac{3(b+v)}{4}-v+1\right]} G\left(\left[\frac{b-v}{2}\right]+\nu, s\right) .
\end{aligned}
$$

We use Lemma 2.6 to establish the existence of positive solutions to the FBVP (1). To this end, one or several of the following conditions will be needed.

(H) $f:[v-2, v-1, \ldots, b+v]_{\mathbb{N}_{v-2}} \times[0,+\infty) \rightarrow[0,+\infty)$ is continuous.

$\left(\mathrm{H}_{1}\right)$ There is a number $p>0$ such that $f(t, y)<A p$ for $0 \leq y \leq p$ and $v-2 \leq t \leq b+v$.

$\left(\mathrm{H}_{2}\right)$ There is a number $p>0$ such that $f(t, y)>B p$ for $\frac{1}{4} p \leq y \leq p$ and $\frac{b+v}{4} \leq t \leq \frac{3(b+v)}{4}$.

$\left(\mathrm{H}_{3}\right) f_{0}>B, f_{\infty}>B$.

$\left(\mathrm{H}_{4}\right) f^{0}<A, f^{\infty}<A$.

$\left(\mathrm{H}_{5}\right) f_{0}>B, f^{\infty}<A$.

$\left(\mathrm{H}_{6}\right) f^{0}<A, f_{\infty}>B$.

$\left(\mathrm{H}_{3}^{*}\right) f_{0}=+\infty, f_{\infty}=+\infty$.

$\left(\mathrm{H}_{4}^{*}\right) f^{0}=0, f^{\infty}=0$.

Let

$$
\mathcal{B}=\left\{y:[v-3, b+v]_{\mathbb{N}_{v-3}} \rightarrow \mathbb{R}, y(v-3)=\Delta y(b+v)=\triangle^{2} y(v-3)=0\right\} .
$$

Then $\mathcal{B}$ is a Banach space with respect to the norm $\|y\|=\max _{t \in[v-3, b+v]_{\mathbb{N}_{v-3}}}|y(t)|$. We define a cone in $\mathcal{B}$ by

$$
\mathcal{K}=\left\{y \in \mathcal{B}: y(t) \geq 0, \min _{\frac{b+v}{4} \leq t \leq \frac{3(b+v)}{4}} y(t) \geq \frac{1}{4}\|y\|\right\} .
$$

Now consider the operator $T$ defined by

$$
(T y)(t)=\sum_{s=0}^{b+1} G(t, s) f(s+v-1, y(s+v-1)) .
$$

It is easy to see that $y=y(t)$ is a solution of the FBVP (1) if and only if $y=y(t)$ is a fixed point of $T$. We shall obtain sufficient conditions for the existence of fixed points of $T$. First, we notice that $T$ is a summation operator on a discrete finite set. Hence, $T$ is trivially completely continuous. From (6), then

$$
\begin{aligned}
\min _{\frac{b+v}{4} \leq t \leq \frac{3(b+v)}{4}}(T y)(t) & \geq \frac{1}{4} \sum_{s=0}^{b+1} G(b+v, s) f(s+v-1, y(s+v-1)) \\
& \geq \frac{1}{4} \max _{t \in[v-2, b+v]_{\mathbb{N}_{v-2}}} \sum_{s=0}^{b+1} G(t, s) f(s+v-1, y(s+v-1)) \\
& =\frac{1}{4}\|T y\|,
\end{aligned}
$$

hence $T \mathcal{K} \subset \mathcal{K}$.

In the sequel, let $\Omega_{\lambda}=\{y \in \mathcal{K}:\|y\|<\lambda\}$, for $\lambda>0, \partial \Omega_{\lambda}=\{y \in \mathcal{K}:\|y\|=\lambda\}$. 
Theorem 3.1 Assume that there exist two different positive numbers $r$ and $R$ such that $f$ satisfies condition $\left(\mathrm{H}_{1}\right)$ at $r$ and condition $\left(\mathrm{H}_{2}\right)$ at $R$. Then the FBVP (1) has at least one positive solution $y_{0} \in \mathcal{K}$ satisfying $\min \{r, R\} \leq\left\|y_{0}\right\| \leq \max \{r, R\}$.

Proof We know that $T: \mathcal{K} \rightarrow \mathcal{K}$, and $T$ is completely continuous. Without loss of generality suppose that $r<R$. Note that for $y \in \partial \Omega_{r}$, we have $\|y\|=r$, so that condition $\left(\mathrm{H}_{1}\right)$ holds for all $y \in \partial \Omega_{r}$. Then

$$
\begin{aligned}
(T y)(t) & \leq \sum_{s=0}^{b+1} G(b+v, s) f(s+v-1, y(s+v-1)) \\
& \leq A r \sum_{s=0}^{b+1} G(b+v, s) \\
& =r
\end{aligned}
$$

i.e., we have $\|T y\| \leq\|y\|$ for $y \in \mathcal{K} \cap \partial \Omega_{r}$.

Note that for $y \in \partial \Omega_{R}$, we have $\|y\|=R$, so condition $\left(\mathrm{H}_{2}\right)$ holds for all $y \in \partial \Omega_{R}$. Since $\left[\frac{b-v}{2}\right]+v \in\left[\frac{b+v}{4}, \frac{3(b+v)}{4}\right]$,

$$
\begin{aligned}
(T y)\left(\left[\frac{b-v}{2}\right]+v\right) & =\sum_{s=0}^{b+1} G\left(\left[\frac{b-v}{2}\right]+v, s\right) f(s+v-1, y(s+v-1)) \\
& \geq \frac{1}{4} \sum_{s=\left[\frac{\left.\frac{b+v}{4}-v+1\right]}{4}-v+1\right]}^{\left[\frac{(b+v)}{2}\right.} G\left(\left[\frac{b-v}{2}\right]+v, s\right) f(s+v-1, y(s+v-1)) \\
& \geq \frac{B R}{4} \sum_{s=\left[\frac{b+v}{4}-v+1\right]}^{\left[\frac{3(b+v)}{4}-v+1\right]} G\left(\left[\frac{b-v}{2}\right]+v, s\right) \\
& =R,
\end{aligned}
$$

i.e., we have $\|T y\| \geq\|y\|$ for $y \in \mathcal{K} \cap \partial \Omega_{R}$.

By Lemma 2.6 that $T$ has at least one fixed point, say, $y_{0} \in \bar{\Omega}_{R} \backslash \Omega_{r}$. This function $y_{0}(t)$ is a positive solution of (1) and satisfies $r \leq\left\|y_{0}\right\| \leq R$. The proof is complete.

Theorem 3.2 Assume that conditions $(\mathrm{H}),\left(\mathrm{H}_{1}\right)$, and $\left(\mathrm{H}_{3}\right)$ hold. Then the FBVP (1) has at least two positive solutions $y_{1}$ and $y_{2}$ with $0<\left\|y_{1}\right\|<p \leq\left\|y_{2}\right\|$.

Proof Suppose that $\left(\mathrm{H}_{3}\right)$ holds. Since $f_{0}>B$, there exist $\varepsilon>0$ and $0<r_{0}<p$ such that

$$
f(t, y) \geq(B+\varepsilon) y, \quad 0 \leq y \leq r_{0}, t \in[v-2, b+v]_{\mathbb{N}_{v-2}} .
$$

Let $r_{1} \in\left(0, r_{0}\right)$. Thus for $y \in \partial \Omega_{r_{1}}$, we have

$$
\begin{aligned}
(T y)\left(\left[\frac{b-v}{2}\right]+v\right) & \geq \sum_{s=0}^{b+1} G\left(\left[\frac{b-v}{2}\right]+v, s\right)(B+\varepsilon) y \\
& \geq(B+\varepsilon) \cdot \frac{1}{4}\|y\| \sum_{s=\left[\frac{b+v}{4}-v+1\right]}^{\left[\frac{3(b+v)}{4}-v+1\right]} G\left(\left[\frac{b-v}{2}\right]+v, s\right)
\end{aligned}
$$




$$
\begin{aligned}
& >B \cdot \frac{1}{4}\|y\| \sum_{s=\left[\frac{b+v}{4}-v+1\right]}^{\left[\frac{3(b+v)}{4}-v+1\right]} G\left(\left[\frac{b-v}{2}\right]+v, s\right) \\
& =r_{1},
\end{aligned}
$$

from which we see that $\|T y\|>\|y\|$ for $y \in \mathcal{K} \cap \partial \Omega_{r_{1}}$.

On the other hand, since $f_{\infty}>B$, there exist $\eta>0$ and $R_{0}>0$ such that

$$
f(t, y) \geq(B+\eta) y, \quad y \geq R_{0}, t \in[v-2, b+v]_{\mathbb{N}_{\nu-2}} .
$$

Choose $R_{1}>\max \left\{4 R_{0}, p\right\}$. For $y \in \partial \Omega_{R_{1}}$, since $y(t) \geq \frac{1}{4}\|y\|>R_{0}$ for $\frac{b+v}{4} \leq t \leq \frac{3(b+v)}{4}$, we have

$$
\begin{aligned}
(T y)\left(\left[\frac{b-v}{2}\right]+v\right) & \geq \sum_{s=0}^{b+1} G\left(\left[\frac{b-v}{2}\right]+v, s\right)(B+\eta) y \\
& \geq(B+\eta) \cdot \frac{1}{4}\|y\| \sum_{s=\left[\frac{b+v}{4}-v+1\right]}^{\left[\frac{3(b+v)}{4}-v+1\right]} G\left(\left[\frac{b-v}{2}\right]+v, s\right) \\
& >B \cdot \frac{1}{4}\|y\| \sum_{s=\left[\frac{b+v}{4}-v+1\right]}^{\left[\frac{3(b+v)}{4}-v+1\right]} G\left(\left[\frac{b-v}{2}\right]+v, s\right) \\
& =R_{1},
\end{aligned}
$$

from which we see that $\|T y\|>\|y\|$ for $y \in \mathcal{K} \cap \partial \Omega_{R_{1}}$.

For any $y \in \partial \Omega_{p}$, from $\left(\mathrm{H}_{1}\right)$, we have $f(t, y)<A p, t \in[v-2, b+v]_{\mathbb{N}_{\nu-2}}$, then

$$
\begin{aligned}
(T y)(t) & =\sum_{s=0}^{b+1} G(t, s) f(s+v-1, y(s+v-1)) \\
& \leq \sum_{s=0}^{b+1} G(b+v, s) A p \\
& =p
\end{aligned}
$$

from which we see that $\|T y\| \leq\|y\|$ for $y \in \mathcal{K} \cap \partial \Omega_{p}$.

Therefore, by Lemma 2.6, we complete the proof.

By the proof of Theorem 3.2, we obtain the following corollary.

Corollary 3.1 Assume that $(\mathrm{H})$ and $\left(\mathrm{H}_{1}\right)$ hold, $\left(\mathrm{H}_{3}\right)$ is replaced by $\left(\mathrm{H}_{3}^{*}\right)$. Then the FBVP (1) has at least two positive solutions $y_{1}$ and $y_{2}$ with $0<\left\|y_{1}\right\|<p \leq\left\|y_{2}\right\|$.

Theorem 3.3 Suppose that conditions $(\mathrm{H}),\left(\mathrm{H}_{2}\right)$, and $\left(\mathrm{H}_{4}\right)$ hold, $f>0$ for $t \in[v-2$, $b+v]_{\mathbb{N}_{v-2}}$. Then the FBVP (1) has at least two positive solutions $y_{1}$ and $y_{2}$ with $0<\left\|y_{1}\right\|<$ $p<\left\|y_{2}\right\|$.

Proof Suppose that $\left(\mathrm{H}_{4}\right)$ holds. Since $f^{0}<A$, one can find $\varepsilon>0(\varepsilon<A)$ and $0<r_{0}<p$ such that

$$
f(t, y) \leq(A-\varepsilon) y, \quad 0 \leq y \leq r_{0}, t \in[v-2, b+v]_{\mathbb{N}_{\nu-2}} .
$$


Let $r_{2} \in\left(0, r_{0}\right)$, for $y \in \partial \Omega_{r_{2}}$, we get

$$
\begin{aligned}
(T y)(t) & =\sum_{s=0}^{b+1} G(t, s) f(s+v-1, y(s+v-1)) \\
& \leq \sum_{s=0}^{b+1} G(b+v, s)(A-\varepsilon) r_{2} \\
& <A r_{2} \sum_{s=0}^{b+1} G(b+v, s) \\
& =r_{2},
\end{aligned}
$$

from which we see that $\|T y\|<\|y\|$ for $y \in \partial \Omega_{r_{2}}$.

On the other hand, since $f^{\infty}<A$, there exist $0<\sigma<A$ and $R_{0}>0$ such that

$$
f(t, y) \leq \sigma y, \quad y \geq R_{0}, t \in[v-2, b+v]_{\mathbb{N}_{\nu-2}} .
$$

Let $M=\max _{(t, y) \in[v-2, b+v] \times\left[0, R_{0}\right]} f(t, y)$, then $0 \leq f(t, y) \leq \sigma y+M, 0 \leq y<+\infty$. Let $R_{2}>$ $\max \left\{p, \frac{M}{A-\sigma}\right\}$, for $y \in \partial \Omega_{R_{2}}$, we have

$$
\begin{aligned}
\|T y\| & \leq \sum_{s=0}^{b+1} G(b+v, s) f(s+v-1, y(s+v-1)) \\
& \leq(\sigma\|y\|+M) \sum_{s=0}^{b+1} G(b+v, s) \\
& =\left(\sigma R_{2}+M\right) \cdot \frac{1}{A} \\
& <R_{2} .
\end{aligned}
$$

Therefore, we have $\|T y\| \leq\|y\|$ for $y \in \partial \Omega_{R_{2}}$.

Finally, for any $y \in \partial \Omega_{p}$, since $\frac{1}{4} p \leq y(t) \leq p$ for $\frac{b+v}{4} \leq t \leq \frac{3(b+v)}{4}$, we have

$$
\begin{aligned}
(T y)\left(\left[\frac{b-v}{2}\right]+v\right) & =\sum_{s=0}^{b+1} G\left(\left[\frac{b-v}{2}\right]+v, s\right) f(s+v-1, y(s+v-1)) \\
& >B \cdot \frac{1}{4} p \sum_{s=\left[\frac{b+v}{4}-v+1\right]}^{\left[\frac{3(b+v)}{4}-v+1\right]} G\left(\left[\frac{b-v}{2}\right]+v, s\right) \\
& =p=\|y\|,
\end{aligned}
$$

from which we see that $\|T y\|>\|y\|$ for $y \in \mathcal{K} \cap \partial \Omega_{p}$.

By Lemma 2.6, the proof is complete.

From the proof of Theorem 3.3, we get the following corollary.

Corollary 3.2 Assume that $(\mathrm{H})$ and $\left(\mathrm{H}_{2}\right)$ hold, $\left(\mathrm{H}_{4}\right)$ is replaced by $\left(\mathrm{H}_{4}^{*}\right)$. Then the FBVP (1) has at least two positive solutions. 
From the proof of Theorem 3.2 and 3.3, we get some theorems and corollaries.

Theorem 3.4 Suppose that condition $(\mathrm{H})$ and $\left(\mathrm{H}_{5}\right)$ are satisfied. Then the FBVP (1) has at least one positive solution.

Corollary 3.3 Suppose that $(\mathrm{H})$ holds. Also assume that $f_{0}=+\infty, f^{\infty}=0$. Then the FBVP (1) has at least one positive solution.

Theorem 3.5 Suppose that $(\mathrm{H})$ and $\left(\mathrm{H}_{6}\right)$ hold. Then the FBVP (1) has at least one positive solution.

Corollary 3.4 Suppose that $(\mathrm{H})$ holds. Also assume that $f^{0}=0, f_{\infty}=+\infty$. Then the FBVP (1) has at least one positive solution.

\section{Some examples}

In this section, we present some examples to validate our main conclusions. In the following examples, we take $v=\frac{17}{8}$ and $b=19$. A computation shows that $A \approx 0.00273$, $B \approx 0.0255$.

Example 4.1 Consider the following Caputo fractional difference boundary value problem:

$$
\left\{\begin{array}{l}
\triangle_{C}^{\frac{17}{8}} y(t)=-\frac{1}{51} e^{t-20}\left(\frac{1}{5} y^{\frac{1}{2}}\left(t+\frac{9}{8}\right)+\frac{1}{64} y^{\frac{3}{2}}\left(t+\frac{9}{8}\right)\right), \\
y\left(-\frac{7}{8}\right)=\triangle y\left(\frac{169}{8}\right)=\triangle^{2} y\left(-\frac{7}{8}\right)=0
\end{array}\right.
$$

where $f(t, y)=\frac{1}{51} e^{t-\frac{169}{8}}\left(\frac{1}{5} y^{\frac{1}{2}}+\frac{1}{64} y^{\frac{3}{2}}\right)$, thus $f_{0}=f_{\infty}=+\infty$. Taking $p=4$, we get

$$
f(t, y) \leq \frac{1}{51}\left(\frac{1}{5} \cdot 4^{\frac{1}{2}}+\frac{1}{64} \cdot 4^{\frac{3}{2}}\right)=0.010294<A p
$$

for $0 \leq y \leq p$ and $v-2 \leq t \leq b+v$. All conditions in Corollary 3.1 are satisfied. Applying Corollary 3.1, the FBVP (7) has at least two positive solutions $y_{1}$ and $y_{2}$ such that $0<\left\|y_{1}\right\|<$ $4<\left\|y_{2}\right\|$.

Example 4.2 Consider the following boundary value problem:

$$
\left\{\begin{array}{l}
\triangle_{C}^{\frac{17}{8}} y(t)=-20\left(2+\cos \left(t+\frac{9}{8}\right)\right) y^{2}\left(t+\frac{9}{8}\right) e^{-2 y\left(t+\frac{9}{8}\right)}, \\
y\left(-\frac{7}{8}\right)=\Delta y\left(\frac{169}{8}\right)=\triangle^{2} y\left(-\frac{7}{8}\right)=0 .
\end{array}\right.
$$

Let $f(t, y)=20(2+\cos t) y^{2} e^{-2 y}$, then $f^{0}=f^{\infty}=0$. We choose $p=4$, when $\frac{1}{4} p \leq y \leq p$ and $\frac{b+v}{4} \leq t \leq \frac{3(b+v)}{4}$, we get

$$
f(t, y) \geq 20(2-1) p^{2} e^{-2 p} \approx 0.026837 p \geq B p .
$$

All conditions in Corollary 3.2 are satisfied. Thus, the FBVP (8) has at least two positive solutions $y_{1}$ and $y_{2}$ such that $0<\left\|y_{1}\right\|<4<\left\|y_{2}\right\|$. 
Example 4.3 Consider the following boundary value problem:

$$
\left\{\begin{array}{l}
\triangle_{C}^{\frac{17}{8}} y(t)=-\frac{1}{400} y\left(t+\frac{9}{8}\right)\left(1+\frac{10}{1+y^{2}\left(t+\frac{9}{8}\right)}\right), \\
y\left(-\frac{7}{8}\right)=\Delta y\left(\frac{169}{8}\right)=\Delta^{2} y\left(-\frac{7}{8}\right)=0,
\end{array}\right.
$$

where $f(t, y)=\frac{1}{400} y\left(1+\frac{10}{1+y^{2}}\right)$, it is easy to compute that $f^{\infty}=0.0025<A$ and $f_{0}=0.0275>$ $B$, which yields the condition $\left(\mathrm{H}_{5}\right)$. By Theorem 3.4, the FBVP (9) has at least one positive solution.

Example 4.4 Consider the following boundary value problem:

$$
\left\{\begin{array}{l}
\triangle_{C}^{\frac{17}{8}} y(t)=-f(t+v-1, y(t+v-1)), \\
y\left(-\frac{7}{8}\right)=\Delta y\left(\frac{169}{8}\right)=\triangle^{2} y\left(-\frac{7}{8}\right)=0,
\end{array}\right.
$$

where $f(t, y)=\frac{1}{367+t} \cdot \frac{10 y}{10 e^{-2 y}+e^{-y}+1}$, it is easy to compute that $f^{0} \approx 0.002269<A$ and $f_{\infty} \approx$ $0.025765>B$. Thus, the conditions $(\mathrm{H})$ and $\left(\mathrm{H}_{6}\right)$ hold. Applying Theorem 3.5, we can find that the FBVP (10) has at least one positive solution.

Remark If $f(t, y)=h(t) g(y)$, our conclusions hold also.

\section{Competing interests}

The authors declare that they have no competing interests.

\section{Authors' contributions}

$\mathrm{HQ}$ conceived of the study and participated in its design and coordination. SK drafted the manuscript. ZJ participated in the design of the study and the sequence correction. All authors read and approved the final manuscript.

\section{Author details}

'School of Mathematics and Computer Sciences, Shanxi Datong University, Datong, Shanxi 037009, P.R. China.

${ }^{2}$ Department of Mathematics, North University of China, Taiyuan, Shanxi 030051, P.R. China.

\section{Acknowledgements}

The authors are very grateful to the reviewers for their valuable suggestions and useful comments, which led to an improvement of this paper. Project supported by the National Natural Science Foundation of China (Grant No. 11271235) and Shanxi Datong University Institute (2009-Y-15, 2010-B-01, 2013K5) and the Development Foundation of Higher Education Department of Shanxi Province $(20101109,20111020)$.

Received: 7 November 2014 Accepted: 7 January 2015 Published online: 12 February 2015

\section{References}

1. Atici, FM, Sengül, S: Modeling with fractional difference equations. J. Math. Anal. Appl. 369, 1-9 (2010)

2. Atici, FM, Eloe, PW: Discrete fractional calculus with the nabla operator. Electron. J. Qual. Theory Differ. Equ. Spec. Ed. I, 2009, 3 (2009)

3. Atici, FM, Eloe, PW: Initial value problems in discrete fractional calculus. Proc. Am. Math. Soc. 137(3), 981-989 (2009)

4. Abdeljawad, T: On Riemann and Caputo fractional differences. Comput. Math. Appl. (2011). doi:10.1016/j.camwa.2011.03.036

5. Holm, M: Sum and difference compositions in discrete fractional calculus. CUBO 13(3), 153-184 (2011)

6. Atici, FM, Eloe, PW: Two-point boundary value problems for finite fractional difference equations. J. Differ. Equ. Appl. 17(4), 445-456 (2011)

7. Goodrich, CS: Some new existence results for fractional difference equation. Int. J. Dyn. Syst. Differ. Equ. 3, 145-162 (2011)

8. Goodrich, CS: Existence and uniqueness of solutions to a fractional difference equation with nonlocal conditions. Comput. Math. Appl. 61, 191-202 (2011)

9. Goodrich, CS: Existence of a positive solution to a system of discrete fractional boundary value problems. Comput. Math. Appl. 217, 4740-4753 (2011)

10. Goodrich, CS: On a discrete fractional three-point boundary value problem. J. Differ. Equ. Appl. 18, 397-415 (2012)

11. Goodrich, CS: On positive solutions to nonlocal fractional and integer-order difference equations. Appl. Anal. Discrete Math. 5, 122-132 (2011) 
12. Goodrich, CS: On nonlinear boundary conditions satisfying certain asymptotic behavior. Nonlinear Anal. 76, 58-67 (2013)

13. Wu, G-C, Baleanu, D: Discrete fractional logistic map and its chaos. Nonlinear Dyn. 75, $283-287$ (2014)

14. Wu, G-C, Baleanu, D: Chaos synchronization of the discrete fractional logistic map. Signal Process. 102, 96-99 (2014)

15. Bai, Z: Eigenvalue intervals for a class of fractional boundary value problem. Comput. Math. Appl. (2012). doi:10.1016/j.camwa.2012.01.004

16. Guo, DJ: Nonlinear Functional Analysis. Shandong Science and Technology Press, Shandong (1985)

Submit your manuscript to a SpringerOpen ${ }^{\circ}$ journal and benefit from:

- Convenient online submission

Rigorous peer review

- Immediate publication on acceptance

- Open access: articles freely available online

- High visibility within the field

- Retaining the copyright to your article 